\title{
Isolation and characterization of the murine Nanog gene promoter
}

\author{
Da Yong WU*, Zhen YAO \\ Laboratory of Molecular Cell Biology, Laboratory of Stem Cell Biology, Institute of Biochemistry and Cell Biology, Shang- \\ hai Institutes for Biological Sciences, Chinese Academy of Sciences, 320 Yue Yang Road, Shanghai 200031, China.
}

\begin{abstract}
Nanog protein is expressed in the interior cells of compacted morulae and maintained till epiblasts but downregulated by implantation stage. It is also expressed in embryonic stem cells, embryonic carcinoma cells and embryonic germ cells but disappeared in differentiated ES cells. In this study, we have isolated, sequenced, and performed the first characterization of the Nanog promoter. The transcription start sites were mapped by primer extension analysis. Two promoter regions were found upstream the transcription start sites and the expression of major Nanog promoter/ reporter gene construct is abolished in differentiated F9 EC cells as compared to the undifferentiated counterpart. We also showed that a putative octamer motif (ATGCAAAA) is necessary for the major promoter activity. Gel shift and supershift assays showed that Oct-1, Oct-4 and Oct-6 protein selectively bind to the octamer motif.
\end{abstract}

Keywords: Nanog, promoter, F9 EC cells, Oct-1, Oct-4, Oct-6.

\section{INTRODUCTION}

Nanog is a newly found homeodomain gene encoding a polypeptide of 305 residues with a divergent homeodomain similar to those in the NK-2 family [1]. The gene was first reported as ENK (early embryo specific expression NK family) and [2] finally designated as Nanog [3,4].

Expression of Nanog appears in the interior cells of compacted morulae, the future inner cell mass (ICM) of blastocysts, and also appears in epiblast and germ cells. It is downregulated in somatic descendants of the inner cell mass. Recent studies revealed that Nanog is important for self-renewal and maintenance of pluripotency in inner cell mass and embryonic stem cells. For example, ES cells overexpressing Nanog does not require LIF (leukemia inhibitory factor) for the renewal and pluripotency; these cells showed resistance to differentiation agents such as trans-retinoic acid. While Nanog null cells will differentiate into visceral and parietal endoderm cells $[3,4]$.

Previous wok in our lab in order to identify the down-

\footnotetext{
*Correspondence: Da Yong WU

Tel: +86-21-54921366; Fax: +86-21-54921366;

E-mail: dywu@sibs.ac.cn
}

Abbreviations: EMSA (Electrophoretic Mobility Shift Assay); EC (Embryonic Carcinoma); WCE (Whole Cell Extract). stream genes of oct 4 by means of SSH (suppressionsubstractive hybridization) identified a new Homeobox gene, a7, which happened to be the same gene as Nanog. The gene was found to be richly expressed in ES cells, but disappeared in RA (retinoic acid) induced differentiated ES cells [5]. In this report, we tried to elucidate the regulating mechanism of the gene by promoter analysis.

\section{MATERIALS AND METHODS}

Isolation and subcloning of murine Nanog genomic DNA

A mouse strain 129/SvJ ES cell genomic library constructed in a Lambda FIX II vector was screened with $\left[{ }^{32} \mathrm{P}\right]$-labeled probe prepared from the full-length cDNA fragment using plaque hybridization protocol [6]. The clones isolated after the final screen was further confirmed by PCR and each positive DNA size was determined by restriction analysis, and the longest one was digested with NotI, then the insert fragment of $17 \mathrm{~kb}$ was subcloned into pBluescript SKII (+) vector. Positive pBluescript clone was sequenced in both sense and antisense orientations. Numbering of the Nanog promoter sequence has been determined by setting the 5'-nucleotide of the Nanog cDNA sequence reported by Wang et al. as position +1 [2]. The TRANSFAC [7] internet site (http://www.gene-regulation.com) and genomatix software (http://www.genomatix.de) were used in the analysis of the DNA sequence.

Reverse transcription-polymerase chain reaction (RT-PCR)

The total RNA was prepared from cultured undifferentiated and differentiated F9 cells with Trizol reagent according to the manufacturer's instruction (Invitrogen). Then the total RNA was 
reverse transcribed using oligo(dT) as primer and SuperScript ${ }^{\mathrm{TM}}$ II $\mathrm{RNase} \mathrm{H}^{-}$Reverse Transcriptase (Invitrogen). Semi-quantitative PCR was carried out with a pair of primers, each of which resides at different exons of the target gene (Tab.1). The PCR products were directly verified by sequencing.

\section{Mapping of the transcription start sites by primer extension analysis}

Primer extension analysis was performed based on the procedure previously described by Sambrook et al. Briefly, an oligonucleotide complementary to nucleotides +177 to +192 of the Nanog cDNA sequence was 5 -end labeled with $\left[\gamma^{32} \mathrm{P}\right]$. Approximately $10^{5} \mathrm{cpm}$ of labeled primer was incubated with $30 \mu \mathrm{g}$ RNA isolated from undifferentiated F9 EC cells using Trizol isolation kit in a reaction mixture brought to a total volume of $30 \mu 1$ with hybridization buffer $(40 \mathrm{mM}$ PIPES pH 6.4, $1 \mathrm{mM}$ EDTA, $0.4 \mathrm{M} \mathrm{NaCl}$, and $80 \%$ formamide). The reaction mixture was denatured at $85^{\circ} \mathrm{C}$ for $10 \mathrm{~min}$ and allowed to hybridize overnight at $30^{\circ} \mathrm{C}$. The annealed primer was extended by incubating with SuperScript ${ }^{\mathrm{TM}}$ II RNase $\mathrm{H}^{-}$Reverse Transcriptase (Invitrogen) in a $20 \mu \mathrm{l}$ reaction containing $50 \mathrm{mM}$ Tris- $\mathrm{HCl}$, pH 8.0, $75 \mathrm{mM} \mathrm{KCl}, 3 \mathrm{mM} \mathrm{MgCl}{ }_{2}, 10 \mathrm{mM}$ dithiothreitol, 40 units of rRNasin (Promega) and $1 \mathrm{mM} \mathrm{dNTPs}$ at $42^{\circ} \mathrm{C}$ for $1 \mathrm{~h}$. The reaction products were analyzed on an $8 \%$ denaturing polyacrylamide gel. The size of the extension products were determined by a concurrently run sequencing ladder using the above primer and Nanog genomic DNA as template.

\section{Cell culture and transient transfection}

F9 EC cells were maintained in Dulbecco's modified Eagle's medium (DMEM) (Life Technologies) supplemented with 10\% fetal bovine serum (Hyclone). F9 EC cells were seeded at $5 \times 10^{4}$ cells per well in 24 well plates $24 \mathrm{~h}$ prior to transfection. $1 \mu \mathrm{g}$ each of the promoter reporters constructs and $0.02 \mu \mathrm{g}$ pRL-TK (Promega) were co-transfected in duplicate by LipofectAMINE 2000 or LipofectAMINE according to the manufacturer's instructions. Cells were lysed with $100 \mu \mathrm{l}$ passive lysis buffer (Promega) $24 \mathrm{~h}$ after transfection. The cell lysates were vortexed and briefly centrifuged to sediment cell debris. A $20 \mu \mathrm{l}$ aliquot of cell lysate was then assayed for luciferase activities using the Dual Luciferase Reporter (DLR) assay system (Promega) according to the supplier's recommendations. All transfections were repeated three times and shown with representative samples. Plasmid DNA was purified using Qiagen tip-20 column. For transfection of EC-differentiated cells, F9 EC cells were seeded at $1 \times 10^{4}$ cells per well in DMEM containing $10 \%$ FBS and $5 \mathrm{mM}$ retinoic acid (RA) for $72 \mathrm{~h}$ [8]. Cell extracts prepared and luciferase activities determined as described above. For transfection of Cos-7

Tab. 1 Oligonucleotides used in the study

\begin{tabular}{|c|c|c|}
\hline Usage & Oligonucleotides & Sequence \\
\hline \multirow[t]{4}{*}{ RT-PCR } & GAPDH (sense) & ccacagtccatgccatcac \\
\hline & (antisense) & ccaccaccetgttgctgtag \\
\hline & Nanog (sense) & cagccetgattcttctaccag \\
\hline & (antisense) & gatgcgttcaccagatagcc \\
\hline \multirow[t]{4}{*}{ Subclone 5 ' fragment } & $5^{\prime}$ end (sense) & ggtaccgatctttcaccagag \\
\hline & (antisense) & agaaagcagtcttcacac \\
\hline & 3' end (sense) & aacgcgttctgggtcaccttaca \\
\hline & (antisense) & ctcgaggtcagtgtgatggcga \\
\hline Primer extension & & atgtcagtgtgatggcgagggaagg \\
\hline \multirow[t]{3}{*}{ Reporter construct (sense) } & -220 & agagctcagctttccetccetc \\
\hline & -132 & tgaattcacagggctg \\
\hline & -78 & agagctcgggtgggtagggtag \\
\hline \multirow[t]{3}{*}{ (antisense) } & +1 & gctcgaggaaggccaacggctc \\
\hline & +6 & actcgagctatctgaaggccaa \\
\hline & +114 & gaagcttgatcatagaaagaagag \\
\hline \multirow[t]{2}{*}{ Primers for mutant construct } & sense & gtcgactctagacaatgtccatggtgg \\
\hline & antisense & tctagagtcgacagctgtaaggtgacc \\
\hline \multirow[t]{2}{*}{ EMSA } & probe (sense) & acagcttcttttgcattacaatgtccatgg \\
\hline & (antisense) & ccatggacattgtaatgcaaaagaagctgt \\
\hline
\end{tabular}


with expression vectors, $3 \mu \mathrm{g}$ of Oct- 4 or Oct- 6 expression plasmid was used. WCEs were prepared $30 \mathrm{~h}$ after transfection.

\section{Promoter-reporter constructs and site-directed mutagenesis}

The 5'-flanking region $(-4,828 /+190)$ of the mouse Nanog gene was subcloned into the KpnI and XhoI sites of pGL3-Basic luciferase reporter vector (Promega). Briefly, the fragment (-3,794/132) from Nhe I to EcoR I digestion of genomic fragment and a PCR amplified fragment $(-132 /+190)$, were successively cloned into the Nhe I and Xho I sites of pGL3-Basic to create pNANP-3,794/+190. pNANP-4,828/+190 was obtained by inserted an amplified fragment $(-4,828 /-3,794)$ into KpnI and Nhe I sites of pNANP-3,794/ +190. pNANP-823/+190, pNANP-395/+190 and pNANP-270/ +190 were generated by progressive 5 ' end deletions of the promoter segment in pNANP-3,794/+190 using exonuclease III and S1 nuclease (MBI). Briefly, pNANP-3,794/+190 was digested with KpnI and NheI, phenol/chloroform extracted, ethanol-precipitated, followed by digestion with exonuclease, the ends were blunted by Klenow fragment in the presence of dNTPs. The blunted and shortened DNA were then circularized by T4 ligase and used to transform the competent DH5a Escherichia coli cells.

DNA fragments of $(-220 /+6),(-78 /+190),(-78 /+114)$ and $(-$ $132 /+1)$ were amplified by PCR using the pNANP $-4,828 /+190$ construct as a template with primers as shown in Tab. 1 and were cloned into pMD-18T vector (Tarkara) and sequenced. Except for (-132/ +1 ), fragments from all positive clones were subcloned into the pGL3Basic vector to create pNANP-220/+6, pNANP-78/+190 and pNANP-78/+114. Construct pNANP-270/+1 was derived by exchange of EcoRI and Hind III restricted parts between pNANP-270/ +190 and pMD-18T-132/+1.

To create the mutant construct pNANPmOct-1, the putative Oct1 binding sequence was replaced by introducing Sal I and Xba I sites with a method based on bridging-based two round PCR method [9] using the primers shown in Tab. 1.

\section{WCEs or nuclear extract preparation and electrophoretic gel mobility shift assay}

Nuclear proteins were extracted from F9 EC cells and Tera-2 human EC cells using the method of [10]. WCEs were prepared by lysing $5 \times 10^{5}$ transfected Cos- 7 cells in $100 \mu$ of high-salt extraction buffer (400 mM KCl, $20 \mathrm{mM}$ Tris- $\mathrm{HCl}$ [pH 8.0], 20\% [v/v] glycerol, $2 \mathrm{mM}$ dithiothreitol, $1 \mathrm{mM}$ phenylmethylsulfonyl fluoride). Cells were lysed by three cycles of freeze $\left(-70^{\circ} \mathrm{C}\right)$-thaw (ice), and the cellular debris was removed by centrifugation at $12,000 \times \mathrm{g}$ for $15 \mathrm{~min}$ at $4^{\circ} \mathrm{C}$. Complementary oligonucleotides (as shown in Tab. 1) were annealed in annealing buffer (10 mM Tris-Cl, pH 7.5, 1 mM EDTA, $100 \mathrm{mM} \mathrm{NaCl}$ ) and the resulting double-stranded oligodeoxynucleotide (dsODN) probe was end-labeled with $\left[\gamma^{-32} \mathrm{P}\right]$ ATP by T4 polynucleotide kinase (Tarkara). For gel mobility shift assays, $2 \times$ $10^{4} \mathrm{cpm}$ probe was incubated with 2 or $6 \mu \mathrm{g}$ of nuclear extracts or WCEs in a $20 \mu$ volume of binding reaction buffer $(10 \mathrm{mM}$ Tris-Cl, pH 7.5, $50 \mathrm{mM} \mathrm{NaCl}, 1 \mathrm{mM} \mathrm{MgCl}, 0.5 \mathrm{mM}$ EDTA, $5 \%$ glycerol, $0.05 \mathrm{mg} / \mathrm{ml}$ Poly $(\mathrm{dI} / \mathrm{dC})$ ) on ice for $30 \mathrm{~min}$. In supershift experiments, Oct-1 (C-21), Oct-6 (H-13) polyclone antibody (Santa Cruz) or rabbit anti-serum against Oct-4 (prepared by our lab) was incubated with nuclear extracts in a $20 \mu$ volume of binding reaction for $30 \mathrm{~min}$ on ice, followed by incubation with the labeled probe on ice for an additional $30 \mathrm{~min}$. Complexes were separated on $4 \%$ non-denaturing polyacrylamide gels. The gels were subsequently dried and autoradio- graphy was performed.

\section{RESULT}

\section{Isolation of the murine Nanog gene and prelimary analysis of the promoter region}

Although the draft sequences of the mouse genome have been already reported, there may be unknown difference between different mouse lines. Here we employed plaque hybridization method and succeeded in isolating a $17 \mathrm{~kb}$ genomic fragment from 129/SvJ ES cell genomic library for the following work. 5'-flanking region of the mouse Nanog gene is approximately $4.8 \mathrm{~kb}$ (Fig. 1A). Promoter region was analyzed with TRANSFAC and GENOMATIX software for the DNA motifs recognized by the known transcription factors. The sequence of the promoter region $(-270 /+190)$ is $100 \%$ homology to NCBI data (XM132755). Some putative transcriptor binding sites are listed in Fig.1.The promoter region of Nanog gene lacked conventional TATA and CAAT boxes, indicating a TATA less gene.

\section{A

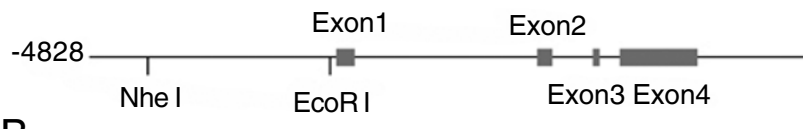 \\ B \\ -229 TGCCCCCTAA GCTTTCCCTC CCTCCCAGTC TGGGTCACCT TACAGCITCT Ap1 Oct1 \\ -179 ITTGCATTAC AATGTCCATG GTGGACCCTG CAGGTGGGAT TAACTGTGAA

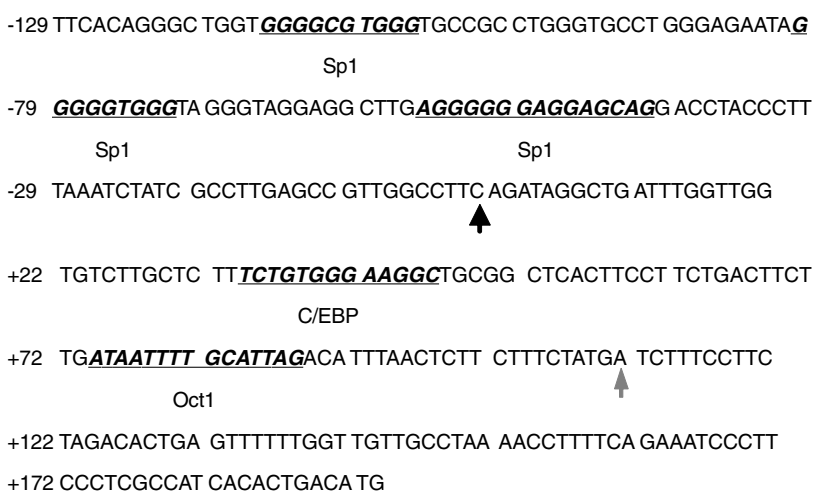

Fig.1 (A) Restriction enzyme sites used in construct and genomic structure of the murine Nanog gene fragment. (B) Sequence of murine Nanog 5'-flanking regions from -229 to +193 . The major site was designated as +1 . Putative transcription factor binding sites (underlined) include: an Ap1 binding site centered -194, two Oct-1 binding sites centered at -176 and +80 , three Sp1 binding sites centered at $-50,-76$ and -110 , a C/EBP binding site centered at +36 . Black triangle represents the major transcription start site. Gray triangle represents the minor one. 


\section{Mapping the transcriptional start sites of the Nanog gene}

The 5' end of Nanog cDNA sequence reported by Wang et al. is located $190 \mathrm{bp}$ upstream of translation site. However, it is necessary to demonstrate whether the site represents the primary start site for Nanog transcription or the gene utilizes other sites of transcription initiation. To map the transcription initiation sites of the Nanog gene, a primer extension analysis was performed using a $\left[{ }^{32} \mathrm{P}\right]-$ labeled 25bp antisense oligonucleotide, which enabled reverse transcription of RNA from F9 EC cells. The analy-

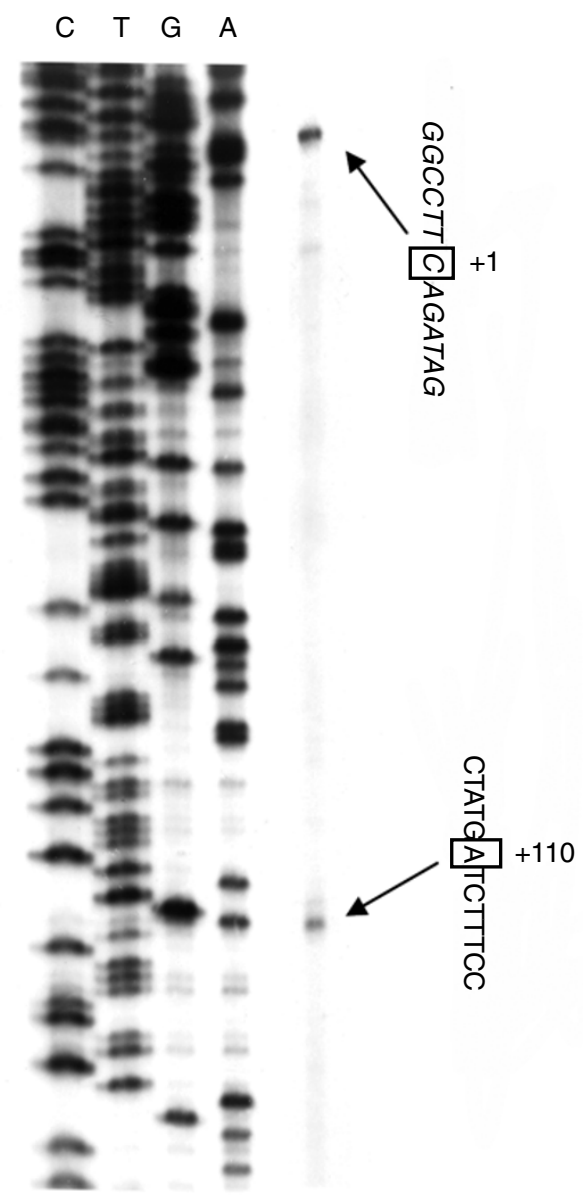

Fig. 2 Primer extension analysis to identify Nanog transcription start sites. $30 \mu \mathrm{g}$ of RNA isolated from F9 EC cells was hybridized to the radiolabeled antisense primer complementary to nucleotides +177 to +201 of the Nanog cDNA sequence and a primer extension reaction was performed using SuperScript ${ }^{\mathrm{TM}}$ II RNase $\mathrm{H}^{-}$Reverse Transcriptase as described in "Methods and materials". The resulting cDNA product was electrophoresed on an $8 \%$ polyacrylamide 7 $\mathrm{M}$ urea gel with a concurrently run sequencing ladder using the above primer. The major site was numbered +1 and minor site +110 . This experiment was repeated and similar results were obtained. sis demonstrated 2 bands, and the major band was arbitrarily designated as position +1 (Fig. 2), which corresponds well with the report by Wang et al. [2]. The minor site is $109 \mathrm{bp}$ downstream of the major one.

\section{Characterization of the Nanog promoter}

To identify the regulatory region crucial to transcriptional activity of the gene, promoter activities of various lengths of the 5'-flanking region were measured. The relative luciferase expression driven by each of the constructs was normalized using a vector containing the Renilla gene as an internal standard to adjust for the differences in transfection efficiency. As seen in Fig. 3A, the expression of pNANP-220/+6 is 54-fold above that of the pGL3-basic. The other region $(-78 /+114)$ is able to activate the reporter gene 3.5-fold above that of pGL3-Basic. As the activity of pNANP $-220 /+6$ is completely abolished by further deletion from -220 to -177 , the fragments $-220 /+6$ and $-78 /$ +114 represent two different promoter regions. Deletion of nucleotides around the major transcription site from pNANP-270/+1 reduced its activity from 10-fold to 3fold above that of pGL3-Basic (Fig. 3B), suggesting this sequence may function as initiator (initiator element) [11].

In addition, analysis of upstream fragment revealed both positive and negative regulatory regions. Region $(-1,571 /$ -823) may contain inhibitory sequence, as the expression of pNANP $-1,571 /+190$ is less than half of that of pNANP $-823 /+190$. Positive elements exist in the region $(-4,828 /-$ $3,794)$.

\section{Effect of differentiation on the Nanog promoter con- structs}

When cultured in the presence of retinoic acid, F9 EC cells can differentiate into cells that exhibit the properties of parietal extra-embryonic endoderm [12]. We have compared mRNA samples between parental and differentiated F9 EC cells by RT-PCR and revealed that the expression of Nanog gene become undetectable after differentiation (Fig. 4A). To determine whether the Nanog promoter/reporter gene constructs are differentially regulated, the Nanog promoter/reporter constructs were transfected into F9 EC-differentiated cells and we found that the expression of Nanog reporter driven by pNANP-220/+6 was completely abolished. While the other construct, pNANP $-78 /+114$ still showed activity (Fig. 4B).

\section{Role of a putative octamer motif in the Nanog pro- moter}

Further deletion from 5 ' end of $(-220 /+6)$ to -177 completely abolished the activity of pNANP $-220 /+6$, indicating the region $(-220 /-177)$ is critical for its expression. Sequence analysis by TRANSFAC software revealed an 


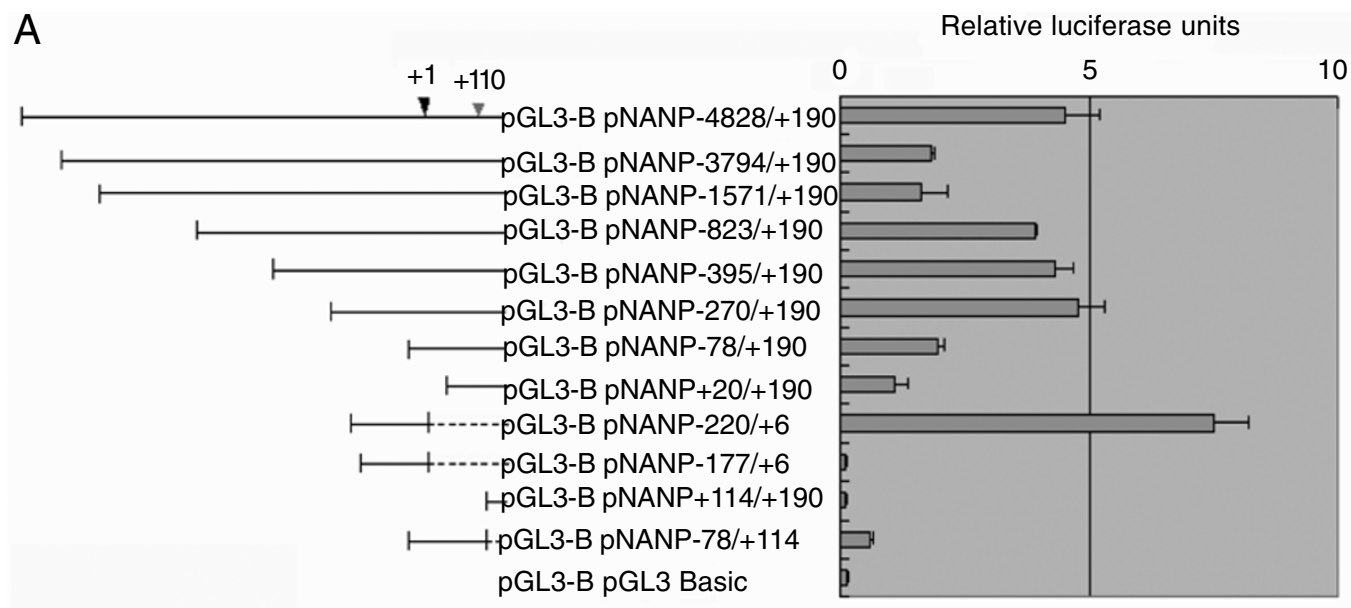

B

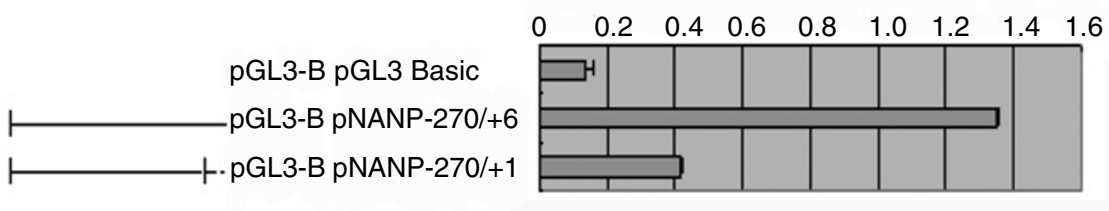

Fig. 3 (A) Analysis of murine Nanog promoter activities in F9 EC cells. Firefly luciferase expression levels were normalized to the luciferase activity of internal Renilla control and expressed as relative luciferase units. The results are the mean \pm S.D. of duplicate from one of three separate experiments. (B) Role of sequence of $(-1 /+6)$ in the activity of pNANP-270/+6.

A

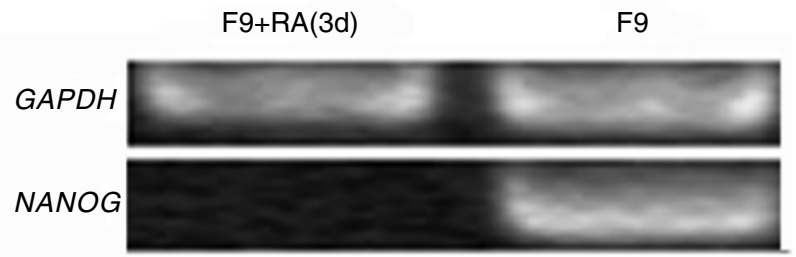

B

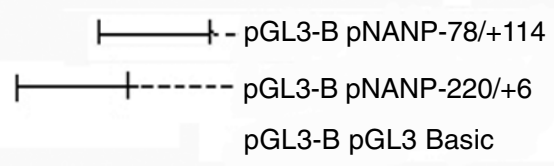

Relative luciferase units

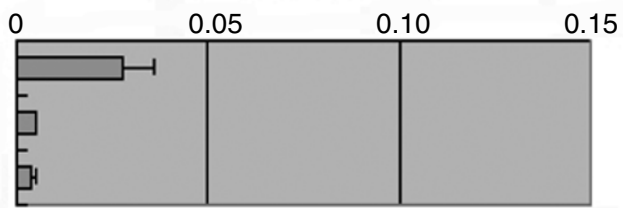

Fig. 4 (A) Expression profile of Nanog gene in differentiated and undifferentiated F9 EC cells RT-PCR was performed using RNA from differentiated and undifferentiated F9 EC cells. (B) Analysis of murine Nanog promoter activities in differentiated F9 EC cells. Firefly luciferase expression levels were normalized to the luciferase activity of internal Renilla control and expressed as relative luciferase units. The results are the mean \pm S.D. of duplicate from one of three separate experiments.

Oct-1 binding site, whose mutation by replacing the original 12 nucleotides with Sal I and Xba I restriction enzyme sites that lack any known cis-element reduced the expres- sion to a basal level (Fig. 5). This result argues strongly that the octamer motif present in the Nanog promoter plays a functional role in the transcription of this gene in undif- 


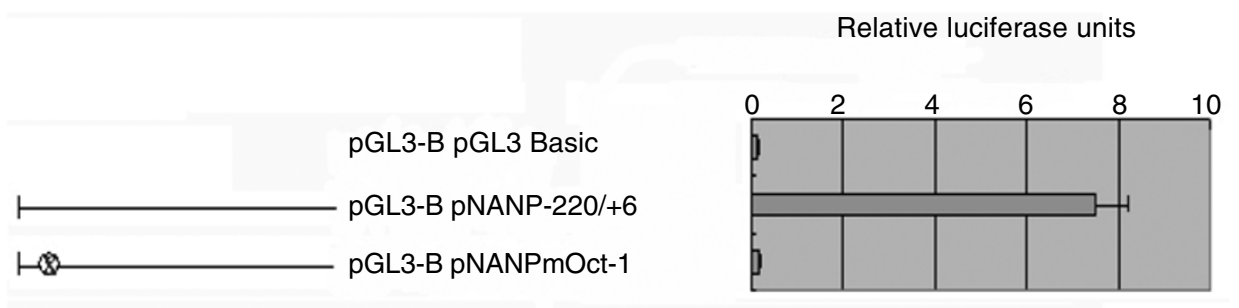

Fig. 5 Role of the Oct-1 binding motif in the expression of the Nanog promoter in EC cells. Duplicate plates of F9 EC cells were transfected with either the wild-type or mutagenized Nanog promoter/reporter construction (left) and analyzed for promoter activity. Firefly luciferase expression levels were normalized to the luciferase activity of internal Renilla control and expressed as relative luciferase units. The results are the mean \pm S.D. of duplicate from one of three separate experiments.

ferentiated F9 EC cells.

The octamer motif present in the Nanog promoter binds the transcription factors Oct-1, Oct-4 and Oct-6

To identify any nuclear proteins associated with the first positive regulatory element $(-183 /-168)$, gel shift assay was performed as described in the method using a doublestranded $\left[{ }^{32} \mathrm{P}\right]$-labeled oligonucleotide containing the sequence for the positive regulatory element. The reaction mixture was then electrophoresed on a polyacrylamide gel and viewed by autoradiography. Previous report indicates that Oct-1 and Oct-4 bind the same site in ES cells [13]. To confirm the existence of above two factors in the DNA/ protein complex, the labeled wild-type Nanog oligonucleotide was incubated with nuclear extract in the presence of antibody. Addition of the Oct-4 antibody resulted in disruption of the complex corresponding to the fastest band and appearance of a supershift band (Fig. 6A, lane 1) indicats that the fastest band contains Oct-4, which was further confirmed by gel shift using WCEs of Oct-4 expressing vector transfected Cos-7 cells (Fig. 6B, lane 2). As the $\mathrm{C}$ terminal epitope of Oct- 1 in mouse is different from that of human [15], addition of the antibody against human Oct-1 protein resulted no change with proteins from F9 cell, but the co-migrating band was identified to be Oct-1 complex from Tera-2 human EC nuclear proteins (Fig. 6A, lane 4), indicating the slowest band contains Oct1 as previous reports [14,15]. Oct-6 is also reported to be expressed in F9 cells [14]. Although the corresponding band is too weak for supershift experiment with nuclear extract (Fig. 6A, lane 5), we detected Oct-6 complex using WCEs from Oct-6 expressing plasmid transfected Cos-7 cells (Fig. 6B, lane 1). However, the identity of the migrating band between Oct- 1 and Oct- 6 is not clear.

\section{DISCUSSION}

Nanog is a critical transcription factor in the orchestra regulating the pluripotency of ES cells [16]. To better un-
A
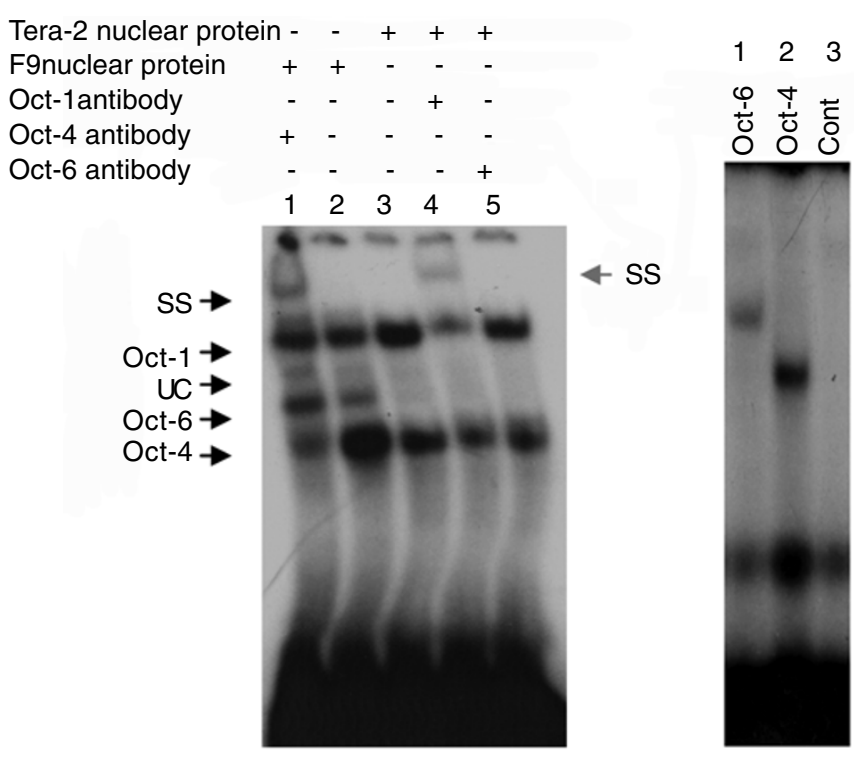

Fig. 6 Gel shift analyses of nuclear proteins or WCEs binding to the Oct-1 probe. Protein-DNA complexes were resolved on $4 \%$ nondenaturing polyacrylamide gel and visualized using autoradiography. (A) Lane 1, probe incubated with F9 nuclear extract proteins in the presence of Oct-4 antibody; lane 2, Positive control with F9 nuclear extract proteins; lanes 3, Positive control with Tera-2 nuclear extract proteins; lane 4 and 5, probe incubated with Tera-2 nuclear extract proteins in the presence of Oct-1 and Oct- 6 antibody respectively; SS with black arrowhead represents Oct-4 supershift; SS with gray arrowhead represents Oct-1 supershift; UC represents unidentified complex. (B) Lane 1, probe incubated with WCEs of pCDNA-oct-6 transfected Cos-7 cells; lane 2, probe incubated with WCEs of pCDNA-oct-4 transfected Cos-7 cells; Lane 3, negative control.

derstand the transcriptional regulation of the Nanog gene we isolated and characterized Nanog gene promoter in this report. By primer extension analysis, we identified two start sites, separated by 109 nucleotides. The major one 
corresponds to the $5^{\prime}$ base of Nanog cDNA sequence reported by Wang et al. and was designated as +1 . The 4.8 $\mathrm{kb}$ of the 5 '-flanking region upstream of the transcription start site was used to generate a panel of Nanog promoter/ reporter gene constructs. This resulted in the identification of two positive regulatory regions present from -220 to +190 . Further experiments indicated that, as expected, the first regulatory region ceased to work in differentiated F9 EC cells. But the other region still showed activity. As Nanog gene is differentially regulated at transcription level and Nanog mRNA become undetectable following EC cell differentiation, there should be some upstream elements or some mechanism to silence its activity in differentiated F9 EC cells.

Sequence $(-1 /+6)$ seems to play as an initiator, defined as a discrete core promoter element that can be functionally similar to the TATA box but operate independently of a TATA box, and the deletion of which may compromise the efficiency of transcription. The functional consensus of initiator is PyCA(+1)NTPyPy [17] in mammal. In this study, however, the start site began at $\mathrm{C}$, and the first nucleotide is A instead of a pyrimidine. But construct lack of the sequence significantly decreased in activity, implying that it may act as an initiator.

The Oct- 1 binding site at -190 is important for the activity of the major promoter, which is confirmed by using deletion and mutagenesis analysis. Gel shift assay demonstrated that the site could bind Oct-1, Oct- 4 and Oct- 6 . Among them, Oct-1 is expressed in all kinds of cells, while expression of Oct- 4 and Oct- 6 are differentially regulated. Oct-4 is abundant in undifferentiated F9 cells but decreased to levels below detection as the cells differentiated [18]. It plays a key role in keeping pluripotency in early embryo development [21]. Oct-6 was originally defined as an embryonic stem cell specific octamer binding factor[14] and it may function as a positive as well as a negative regulator of transcription depending on the exact promoter architecture $[19,20]$. Previous work in our lab, which showed that over-expression of oct-4 could maintain the expression of Nanog gene and became resisted to RA induced differentiation, together with gel shift result, may suggest Oct-4 being responsible for Nanog gene transcription. It was previously reported that Oct-4 often activates promoter in the presence of co-activator Sox-2 binding near that of octamer motif [21]. However in this case, we failed to detect Sox-2 complex by gel shift (data not shown). Our unshown data indicate that transfection of oct-4 or oct-6 expression vector only could not enhance Nanog expression, thus the activation mechanism remains to be elucidated.

Unlike the upstream Oct- 1 binding site, the site at +80 has no effect on promoter activity (not shown). There are also some other putative elements listed in Fig. 1, but it is still obscure whether these elements are necessary for the Nanog gene expression. Mechanism such as methylation recently was reported to couple with the silencing the Nanog gene expression [22]. As Nanog expression complies well with the methylation process in early development [23], it might also be necessary to search for the methylation status of above possible binding sites.

Taken together, in the initial characterization of the promoter of the murine Nanog gene, two transcription start sites were detected and two promoter regions were established; An Octamer motif is important for the expression of Nanog gene in the F9 cells and transcription factors Oct-1, Oct-4 and Oct-6 bind the site. As Nanog is a crucial factor in maintaining the pluripotency of ICM and ES cells, it deserves further exploration on its expression regulation.

\section{ACKNOWLEDGEMENT}

We thank Professor Jing De ZHU and Stephen T. SMALE for helpful discussions on the work and Prof. Angie RIZZINO for Sox-2 expression plasmid. We also thank Mrs. Xiulan LI and Mr. Lin Ping BAO in our lab for their technical supports and other kind helps.

Received, Mar 5, 2005

Revised, Mar 12, 2005

Accepted, Mar 21, 2005

\section{REFERENCES}

1 Harvey RP. Nk-2 homeobox genes and heart development. Dev Biol 1996; 178:203-16.

2 Wang SH, Tsai MS, Chiang MF, Li H. A novel NK-type homeobox gene, ENK (early embryo specific NK), preferentially expressed in embryonic stem cells. Gene Expr Patterns 2003; 3:99-103.

3 Chambers I, Colby D, Robertson M, et al. Functional expression cloning of Nanog, a pluripotency sustaining factor in embryonic stem cells. Cell 2003; 113:643-55.

4 Mitsui K, Tokuzawa Y, Itoh H, et al. The homeoprotein Nanog is required for maintenance of pluripotency in mouse epiblast and ES cells. Cell 2003; 113:631-42.

5 ZW Du, HC Cong, Z Yao. Identification of putative downstream genes for oct-4 by suppression-subtractive hybridization. Biochem Biophys Res Commun 2001; 282:701-6.

6 Sambrook J, Fritsch EF, Maniatis T. Molecular Cloning: A Laboratory Manual. 2nd edition. Cold Spring Harbor Laboratory Press, New York 1989:185-7.

7 Wingender E, Chen X, Hehl R, et al. TRANSFAC: an integrated system for gene expression regulation. Nucleic Acids Res 20001; 28:316-9.

8 Wiebe MS, Wilder PJ, Kelly D, Rizzino A. Isolation, characterization, and differential expression of the murine Sox-2 promoter. Gene 2000; 246:383-93.

9 Xu J, Zhu JD, Ni M, Wan F, Gu JR. The ATF/CREB site is the key element for transcription of the human RNA methyltrans- 
ferase like 1(RNMTL1) gene, a newly discovered 17p13.3 gene. Cell Res 2002; 12:177-97.

10 Andrews PA, Jones JA. A rapid micropreparation technique for extraction of DNA-binding proteins from limiting numbers of mammalian cells. Nucleic Acids Res 1991; 19:2499.

11 Smale ST, Baltimore D. The "initiator" as a transcription control element. Cell 1989; 57:103-13.

12 Strickland S, Mahdavi V. The induction of differentiation in teratocarcinoma stem cells by retinoic acid. Cell 1978; 15:393-403.

13 Schoorlemmer J, Kruijer W. Octamer-dependent regulation of the kFGF gene in embryonic carcinoma and embryonic stem cells. Mech Dev 1991; 36:75-86.

14 Scholer HR, Hatzopoulos AK, Balling R, Suzuki N, Gruss PA. Family of octamer-specific proteins present during mouse embryogenesis: evidence for germline-specific expression of an Oct factor. EMBO J 1989; 8:2543-50.

15 Rosfjord E, Rizzino A. The octamer motif present in the Rex-1 promoter binds Oct-1 and Oct-3 expressed by EC cells and ES cells. Biochem Biophys Res Commun 1994; 203:1795-802.

16 Cavaleri F, Scholer HR. Nanog: a new recruit to the embryonic stem cell orchestra. Cell 2003; 113:551-2.
17 Smale ST. Transcription initiations from TATA-less promoters within eukaryotic protein-coding genes. Biochim Biophys Acta 1997; 1351:73-88.

18 Wang L, Schultz GA. Expression of Oct-4 during differentiation of murine F9 cells. Biochem Cell Biol 1996; 74:579-84.

19 Suzuki N, Rohdewohld H, Neuman T, Gruss P, Scholer HR. Oct-6: a POU transcription factor expressed in embryonal stem cells and in the developing brain.EMBO J 1990; 9:3723-32.

20 Ben-Shushan E, Thompson JR, Gudas LJ, Bergman Y. Rex-1, a gene encoding a transcription factor expressed in the early embryo, is regulated via Oct-3/4 and Oct- 6 binding to an octamer site and a novel protein, Rox-1, binding to an adjacent site. Mol Cell Biol 1998; 18:1866-78.

21 Pan GJ, Chang ZY, Scholer HR, Pei D. Stem cell pluripotency and transcription factor Oct4.Cell Res 2002; 12:321-9.

22 Deb-Rinker P, Ly D, Jezierski A, Sikorska M, Walker PR. Sequential DNA methylation of the Nanog and Oct-4 upstream regions in human NT2 cells during neuronal differentiation. J Biol Chem 2005; 280:6257-60.

$23 \mathrm{Li}$ E. Chromatin modification and epigenetic reprogramming in mammalian development. Nat Rev Genet 2002; 3:662-73. 